\title{
Structural and mechanistic basis for CBP/P300 recruitment to the Notch transcription complex
}

\author{
Ellie Kolb, Daniel Hall, Jordan Webb, and Rhett Kovall \\ Department of Molecular Genetics, Biochemistry, and Microbiology, University of Cincinnati. \\ Cincinnati, OH 45219, kolbem@mail.uc.edu
}

Notch signaling is a highly conserved pathway in metazoans that is crucial for regulating embryonic development and maintaining tissue homeostasis. Misregulation of Notch signaling has been implicated in certain cancers, cardiovascular diseases, and neurodegeneration. During transcriptional activation, the Notch transcription factor CSL (CBF1/RBP-J, Su(H), Lag1) forms a core Notch Ternary Complex (NTC) with NICD (Notch Intracellular Domain) and Maml (Mastermind-like), and then recruits several coactivators, including the histone acetyltransferase enzymes CBP and P300. Maml and NICD binding to CBP/P300 are required to activate transcription; however, the structural basis for formation of these complexes is unknown. Using Electromobility Shift Assays, we have shown that the KIX domain of CBP interacts with both Maml and NICD. Additionally, we have used Isothermal Titration Calorimetry (ITC) to uncover the thermodynamic binding parameters of these complexes and have shown that the KIX domain of CBP interacts with Maml and NICD with micromolar affinity. Furthermore, we have designed fusion protein constructs to promote crystallization of this large Notch transcription complex and have shown that these fusion proteins behave similarly to the native proteins. Preliminary crystallization experiments are underway to solve 1. the KIX domain of CBP bound to Maml, 2. KIX bound to the Ankyrin repeats (ANK) of NICD, and 3. KIX in complex with the NTC. 